\title{
A reappraisal of the quantitative relationship between sugar intake and dental caries: the need for new criteria for developing goals for sugar intake
}

Aubrey Sheiham ${ }^{1 *}$ and W Philip T James ${ }^{2}$

\begin{abstract}
Background: There is a clear relation between sugars and caries. However, no analysis has yet been made of the lifetime burden of caries induced by sugar to see whether the WHO goal of 10\% level is optimum and compatible with low levels of caries. The objective of this study was to re-examine the dose-response and quantitative relationship between sugar intake and the incidence of dental caries and to see whether the WHO goal for sugar intake of $10 \%$ of energy intake (E) is optimum for low levels of caries in children and adults.
\end{abstract}

Methods: Analyses focused on countries where sugar intakes changed because of wartime restrictions or as part of the nutritional transition. A re-analysis of the dose-response relation between dietary sugar and caries incidence in teeth with different levels of susceptibility to dental caries in nationally representative samples of Japanese children. The impact of fluoride on levels of caries was also assessed.

Results: Meticulous Japanese data on caries incidence in two types of teeth show robust log-linear relationships to sugar intakes from 0\%E to 10\%E sugar with a 10 fold increase in caries if caries is assessed over several years' exposure to sugar rather than only for the first year after tooth eruption. Adults aged 65 years and older living in water fluoridated areas where high proportions of people used fluoridated toothpastes, had nearly half of all tooth surfaces affected by caries. This more extensive burden of disease in adults does not occur if sugar intakes are limited to $<3 \%$ energy intake.

Conclusions: There is a robust log-linear relationship of caries to sugar intakes from 0\%E to 10\%E sugar. A 10\%E sugar intake induces a costly burden of caries. These findings imply that public health goals need to set sugar intakes ideally $<3 \%$ E with $<5 \%$ E as a pragmatic goal, even when fluoride is widely used. Adult as well as children's caries burdens should define the new criteria for developing goals for sugar intake.

Keywords: Caries, Sugar, Dose-response, Goals

\section{Background}

Dental caries is the most common of all chronic diseases in industrial and in most lower income countries. "Oral conditions affected 3.9 billion people, and untreated caries in permanent teeth was the most prevalent condition evaluated for the entire Global Burden of Disease (GBD) 2010 Study with a global prevalence of $35 \%$ for all ages

\footnotetext{
*Correspondence: a.sheiham@ucl.ac.uk

${ }^{1}$ Emeritus Professor of Dental Public Health, Department of Epidemiology \&

Public Health, University College London, London, UK

Full list of author information is available at the end of the article
}

combined." [1]. Although the prevalence and severity of dental caries have decreased substantially in the past two decades, this largely preventable disease is still common, increases significantly with age, and remains a public health problem. Sugars are recognised as by far the most important dietary factor in the development of dental caries and there is a clear understanding of the biology of the process of enamel dissolution induced by acid fermented products of sugars by the action of bacteria [2]. A variety of factors modify this effect. They include saliva, the frequency of sugar intakes, the individual tooth 
chosen for examination, as well as the post-eruptive age of the tooth and whether long standing fluoride use has changed the enamel to make it more resistant to acid attack [3]. Despite the use of fluoride and improvements in preventive dentistry, the burden of dental caries remains unacceptably high worldwide, particularly when, in addition to the traditional focus on childhood caries, the caries burden in adults is considered. Given the acceptance that sugar intake is the primary cause of dental caries with variations in the incidence and prevalence reflecting the impact of the above modifying factors, the usual caveats relating potentially to unknown causes do not apply because there is no other mechanism for inducing caries so the only confounding factors i.e. tooth brushing and the use of fluoride in drinking water or toothpaste serve to reduce the magnitude of the simple relationship between sugar intake changes and caries incidence.

The most extensive systematic review of the relationship between caries and sugars was very recently conducted by Moynihan and Kelly [4]. One of the objectives of their review was to update evidence on the association between amount of sugars intake and dental caries, and on the effect of restricting sugars intake to $<10 \%$ and $<5 \%$ energy (E) on caries as $<10 \% \mathrm{E}$ is the current $\mathrm{WHO}$ guideline on sugar intake. They searched the Central Register of Controlled Trials, Latin American and Caribbean Health Sciences, China National Knowledge Infrastructure and Wanfang, S. African Department of Health data. From 5990 papers, they identified 55 studies that were eligible; these were 3 intervention, 8 cohort, 20 population and 24 cross-sectional studies. Forty two of the 50 studies in children and 5/5 studies in adults reported at least one positive association between some measure of daily total, free or added sugars, rather than between frequency of sugar consumption and caries. Moynihan and Kelly [4] found that studies of caries rates when sugars intake was above or $<10 \%$ E provided evidence of 'Moderate' quality in support of lower sugars using the new Grade system which relies heavily on double blind trials even when dealing with public health issues relating to population diets. When considering the lower cut-off $(<5 \% \mathrm{E})$, a significant relationship was observed but the evidence was judged to be of "very low" quality because it was based on detailed observational studies in cohorts undergoing major national dietary change, and not involving a defined randomized intervention group within the society concerned.

Given that the recent systematic review by Moynihan and Kelly [4] showed a clear relation between sugars and caries, another systematic review of this issue is not warranted. However, no analysis has yet been made of the lifetime burden of caries induced by sugar to see whether the WHO goal of $10 \%$ level is optimum and compatible with low levels of caries. Given therefore the renewed interest in the optimum intake for sugar intake now that dietary sugars have been shown to help induce excess weight gain and obesity [5], this paper re-examines the quantitative relationship between sugar intake and the development and the life-long burden of dental caries.

\section{Methods}

Primary information was first obtained from nationally representative prevalence and incidence data on caries. These assessments also contained sugar intakes specified either by dietary surveys or by the national intake assessed from the UN Food and Agriculture Organizations Food Balance Sheet data [6] were derived in a standard way from industrial and other criteria specified by national governments. In addition, personal contacts with international scholars familiar with national data published either as governmental reports or in other formats were obtained and translated. For example, we analyzed data from Japanese reports on national annual examination data of school children, based on nationwide sample surveys published by Ministry of Education and some local surveys kindly provided by colleagues. From these sources we selected data providing data on assessments of non-milk extrinsic sugars intakes from dietary questionnaires or from national data on the annual sugar availability or dietary intake. Sugars in this international context relate to national statistics for sucrose availability but in the US, fructose syrups are included and in the UK the term "non-milk extrinsic sugars" is used to define these non-lactose disaccharides with maltose making a negligible contribution. It is well recognized biologically that the monosaccharides fructose plus glucose in combination can also cause caries, but in practice most of the statistics in these re analyses relate to sucrose intake or that available for consumption. Intakes refer to sugars specifically available for consumption after correction for waste and other non-food uses of sugar. The statistics do not take account of sugars contained in dried fruit. These intakes refer to absolute intakes and not to the frequency of sugar intake during the day. Sugar intakes specified in $\mathrm{kg} / \mathrm{head} / \mathrm{yr}$ were expressed in energy \% by taking the energy as $4 \mathrm{kcal} / \mathrm{g}$ for sugar and a global national average energy intake (including children) of 2,000 kcal/head/day. Particular attention was paid to countries with sugar intakes of $<15 \%$ energy or $<27.5 \mathrm{~kg}$ per annum and then those where detailed examination of the caries prevalence were also monitored. Countries where sugar intakes were previously very low, or had changed due to rationing in wartime or under sanctions were included. Studies fulfilling those criteria were Tristan de Cunha [7], Nigeria [8], Germany [9], Channel Islands [10], Norway [11] Iraq [12] and Japan [13]. 
A re-assessment was carried out of the dose-response relation between dietary sugar and caries incidence in teeth with different levels of susceptibility to dental caries in Japanese children [14] together with an assessment of the extent of continuing caries in adult life. The quantitative impact of fluoride on the prevalence and severity of caries was also then assessed. Three countries, Ireland, Australia and the United States, where water fluoridation had been implemented for over 30 years were selected on the basis that they had a legal requirement for universal water fluoridation, as well as the usual use of fluoride toothpastes [15-17]. Because a comprehensive systematic review, using similar methods has just been published [4] the analogous findings of a proportionally greater caries experience with greater sugar intakes with rising sugar intakes are not reported. Here, we assess the importance of differential tooth susceptibility to caries, the postponement of caries incidence by fluoride and the burden of caries on a life-time basis.

\section{Results}

\section{Secular changes in sugar intake and dental caries}

National analyses from low-income countries show that dental caries was very uncommon before people starting consuming refined sugars. Thus, caries was rare in Tristan de Cunhan in all ages when in 1938 average sugar intakes were only $1.8 \mathrm{~g} /$ day or $0.4 \%$ of average energy intake (E) per person: caries affected $2 \%$ of 13 to $19 \mathrm{yr}$ old adolescents and 7\% of adults aged 30-39 years. However, by the 1960 s sugar intake was $150 \mathrm{~g}$ per day $(30 \% \mathrm{E})$ and $17.5 \%$ of children already had caries [7]. Similarly, Sheiham reported that only $2 \%$ of urban and rural Nigerians of all ages including the elderly, whose permanent teeth had been exposed to diets for half a century, had any dental caries when their sugar intakes were about $2 \mathrm{~g} /$ day per person $(0.4 \% \mathrm{E})[8]$. Thus Sheiham's study indicates that with very low intakes of sugars, caries rarely occurs at any age.

Two cross sectional surveys indicate that there is a strong positive correlation, ranging from 0.72 to 0.95, between sugar consumption and DMFT $[9,13]$. Wartime analyses amplify the close correlation between sugar availability and the prevalence and severity of dental caries as seen in Norway [11], and in Europe generally during and after World War II and more recently in Iraq during the UN sanctions [12].

The most comprehensive national data are from Takeuchi in Japan where before, during and after World War II, per capita sugars levels decreased from $15 \mathrm{~kg}$ per year $(\approx 8.2 \% \mathrm{E})$ to $0.2 \mathrm{~kg}(\approx 0.1 \% \mathrm{E})$ and then increased again to $15 \mathrm{~kg}$ per year over a period of 11 years thereby allowing analysis of the impact of sugar on caries cavitation [13]. In the studies, each tooth was examined separately at yearly intervals in each individual and showed a clear relationship between the average prevailing sugar intake levels and dental caries that had progressed to cavitation.

\section{Dose-response relationships}

The dose-response relationship between sugars and caries is only sigmoid, as previously suggested, if one chooses the most resistant incisor teeth and considers only the first year of exposure to sugar, as shown in Figure 1 [14]. When, however, the more caries susceptible molar teeth are assessed and exposure to sugars for 7-8 years is considered, then the relationship becomes log-linear or, in arithmetic terms, curvilinear (Figure 2) even though the diagnostic level of caries used was cavitation, a late stage of caries [18]. There is no evident threshold for sugars but a log-linear increase in caries rates between $<1 \mathrm{~kg}$ sugar $/ \mathrm{head} / \mathrm{yr} . \quad(\approx 0.05 \% \mathrm{E})$ and $5-7.5 \mathrm{~kg}$ sugar/head/yr. $(\approx 2.7 \%-4.1 \% \mathrm{E})$ if teeth that have been erupted for $7-8$ years are considered $[14,18,19]$. The positive correlation between sugars and caries was +0.7 with a log-linear relation at both lower and higher sugar intake levels for all tooth types if 1-8 years of sugar exposure is considered $[13,14,18,19]$.

The relationship holds for both increases and decreases in sugars consumption which limits the possibility of confounders affecting that relationship. From almost zero intakes on a population basis (with probably modest individual variation in sugar intake) an increase from about zero sugar intake to an average of $5 \mathrm{~kg} / \mathrm{yr}(\approx 2.7 \% \mathrm{E})$ leads to at least a doubling in the prevalence rate of caries in molars, 8 years after tooth eruption (Figure 1).

\section{The impact of fluoride on the sugar-caries relationship}

Fluoride is associated with about 25\% lower caries experience when sugar intakes are constant between $10-15 \% \mathrm{E}$ in 12 year-old children [20]. The widescale use of fluoride toothpaste is a reasonable explanation for the decline in children's caries in many countries since the 1970s, yet what then becomes relatively evident is that caries becomes more prominent in adolescents and adults $[4,21]$.

Ireland has had a mandatory national water fluoridation policy since 1964 but some areas have not implemented the fluoridation policy thereby allowing a comparison within a country where fluoride toothpaste is in widespread use but drinking water fluoride varies. Additional benefits accrued from having fluoride in water as well as toothpastes but $7.3 \%$ of even the youngest adults aged 16-24 years with lifelong fluoride exposure still had dental caries experience in 4.6 teeth as did $53 \%$ of the 35-44-year-olds assessed 35 years after the beginning of water fluoridation: the mean DMFT was 13.3 and 16.0 in those living in non-fluoridated areas [15]. Australia has water fluoridation in a number of cities, 


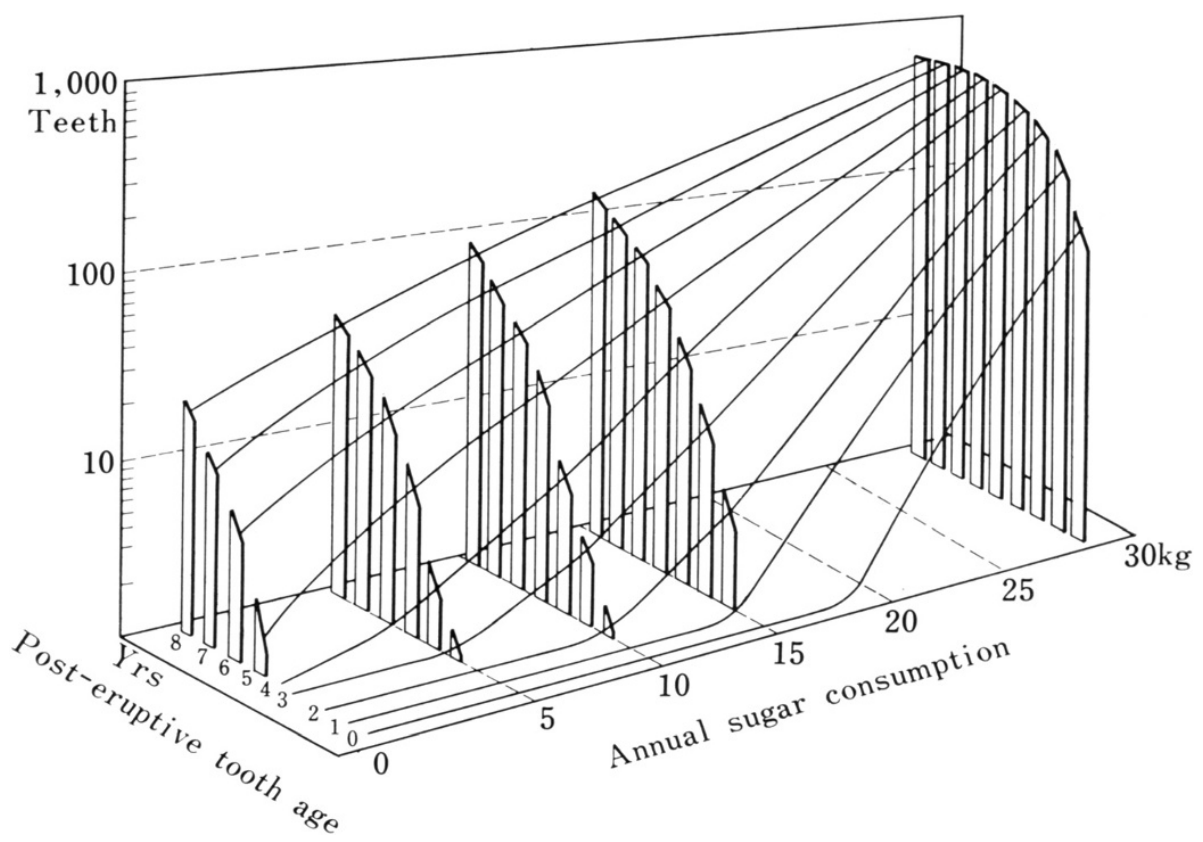

Figure 1 Three-dimensional model of the cumulative numbers of caries in upper central incisor teeth. Data were plotted on a log scale, by post-eruptive tooth age up to 8 years, and related to the average annual sugar consumption per head in Japan from 1935 to 1957 (Takeuchi et al. [14], with permission).

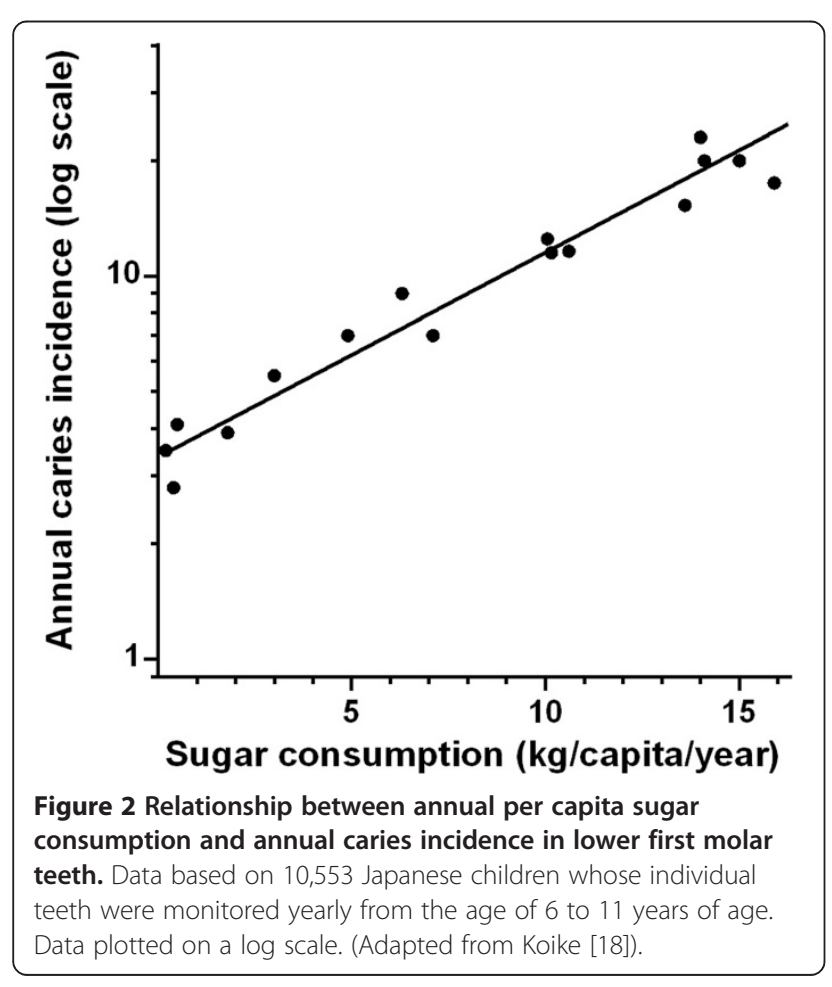

but despite fluoride use from both toothpastes and drinking water the mean DMFT and DF Surfaces for all adults increased; adults aged 65 years and older had ten times higher levels of caries than 15-24-year-olds [16]. Thus although fluoride reduces caries, unacceptably high levels of caries in adults persist in all countries, even in those with widespread water fluoridation and the use of fluoridated toothpastes [21].

National US data show that despite the widescale use of fluoridated toothpaste and consumption of fluoridated water by $66 \%$ of Americans since the 1960 s, $92 \%$ of USA adults aged 20 to 64 have experienced dental caries in their permanent teeth and those aged 65 to 74 years have on average 70 decayed, missing or filled surfaces (DMFS) of the possible surfaces at risk of caries in the permanent teeth (Figure 3) [17].

The majority of caries in permanent teeth occurs in adults, not in children. That is evident in countries with low as well as high sugar intakes irrespective of the fluoride intakes [15-17,21-25]. Thus in China, the mean DMFT was 0.5 in 12-year-olds, 4.0 when $35-44$ years and 14.6 in 65-74-year-olds when the average sugar intake was $6.4 \mathrm{~kg} /$ person/year $(3.5 \% \mathrm{E})$ in 2005 albeit with wellknown intake differences between urban and rural areas [24]. In South Korea with sugar intakes between $7 \cdot 3$ g/day $(1.5 \% \mathrm{E})$ in 1998 and $10.5 \mathrm{~g} /$ day $(2.1 \% \mathrm{E})$ in 2011 , the DMFT in 12 year olds was 2.0 and in $35-44$ year-olds $5 \cdot 2$ in 2011; only 3.2 surfaces were affected in 12 year olds but 14.3 were carious in $35-44$ year olds, and 39 


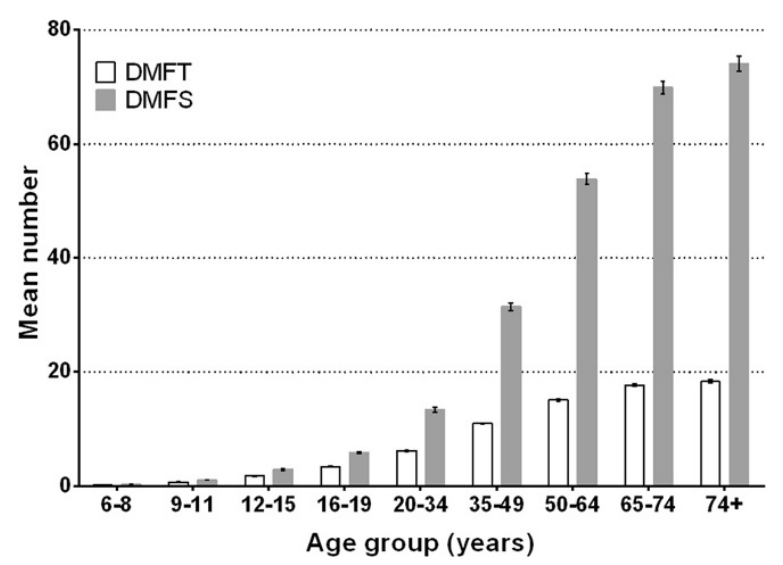

Figure 3 The decayed, missing and filled teeth (DMFT) and filled tooth surfaces (DMFS) in a national USA sample showing that the numbers of DMFS was over 70 by $\mathbf{7 5}$ years. (Adapted from Dye et al. [17]).

surfaces out of a total 160 by $65-74$ years [25]. These data emphasise the importance of considering the adult burden when assessing optimum intakes of sugar.

\section{Discussion}

That dietary sugar causes caries is rarely disputed although some academic dentists emphasise the importance of the frequency of sugar consumption and the adverse effects of sugary drinks and confectionery rather than sugary foods. Each of these factors enhances the short-term induction of caries [2]. Nevertheless, studies which simply deal with sugar intakes predominantly in food, as seen in such unusual settings as the island of Tristan de Cunha, reveal that added sugar, even within food, induces dental caries. Good preventive dental care and the use of fluoridated toothpastes, preferably with fluoridated water supplies to cover the needs of those who do not practice careful personalised preventive measures, is also claimed to be adequate without the need to consider lowering sugar intakes. However, the increasing caries levels as people progress through the life course [26] shows that the major caries burden is in adults, although good preventive dental care and the use of fluoride do reduce caries [20].

\section{The nature of the dose-response relationship of caries development to sugar intakes}

The sugar-caries relationship is log-linear or, in arithmetic terms, curvilinear. Originally we proposed that the dose response relationship corresponded to a sigmoid curve since the Takeuchi data showed low levels of dental caries in upper incisor teeth at sugar intakes below $10 \mathrm{~kg} /$ year (5.5\%E) [13,27]. Greater sugar intakes induced marked increases in new caries lesions and the upper flat part of the curve seemed to reflect a maximum rate of tooth decay implying a sigmoidal response to sugar intakes. This was mistaken for five reasons. First we had, as a very conservative approach, used the least susceptible teeth, the incisors, for assessing threshold sugar intakes. Clearly, from a disease burden point of view the susceptibility of all teeth is important. Second, we followed the traditional and still almost routine approach to dental surveys and analyses by focussing on tooth decay in children because the emphasis has always been on the need for children to be treated. Third, we arbitrarily chose caries occurring within a year of eruption as a good sensitive index which could readily be related to the prevailing sugar intakes when 'in practice' it was already evident that even incisor teeth which were caries-free one year post-eruption, did indeed develop caries as exposure to sugar continued. The spurious concept of a plateau developed simply because it was not possible to document cavitation, the late stage of sugar induced enamel dissolution, within the year even if there were higher levels of sugar intake. Furthermore by choosing this time frame the overall burden of caries, even in children, was markedly underestimated. Then we failed to take account of the fact that although the age-specific rates of caries are lower with the use of fluoride, fluoride use merely delayed the ultimate onset of caries so that adolescents and adults were then the age groups displaying the caries burden. Finally we were slow to realise that the apparent plateau in caries development at higher sugar intakes simply meant that sugar intakes were so high that all tooth surfaces were approaching their sugar saturation level.

The sugar-caries relationship in adults has been largely ignored: all the conclusions on safe levels of sugar and the relationship between sugar and caries are based on children's data. With fluoride and greater dental care caries has declined in children so some dental authorities have concluded that sugars are not a major determinant of caries provided fluoride toothpaste is use diligently with or without water fluoridation. However, it is now evident that the majority of caries occurs in adults, not in children, because the disease is cumulative and the rates of caries in individuals tracks from early childhood to adolescence and then into adulthood $[21,26]$. So the conclusion that sugar is not the major determinant of caries, is simply wrong.

The chronic cumulative impact of caries arising from the continuing availability of susceptible teeth and natural teeth surfaces to long term sugar exposure is vividly shown by a Dunedin, New Zealand, longitudinal study where a comprehensive free school based dental service for children failed to prevent the progressive increase in caries although the rate varied dramatically between individuals followed from 5 years of age for the next 27 years [26]. Although it could be argued that those 
showing a very small increase in caries burden may have been particularly attentive to preventive measures after leaving school, yet on a population basis there is already a marked burden of disease in early adulthood. The data from the US, Sweden and Japan [17,22,23] show that the major burden of sugar induced caries increases markedly after 32 years of age [21].

\section{The economic burden of adult caries}

Caries impacts seriously on individuals and society in terms of pain, discomfort, social and functional limitation and handicap so the effect on the quality of life is considerable [28]. The WHO estimates that oral diseases are the fourth most expensive diseases to treat in most industrialized countries [29]. In some countries such as Germany and Japan, dental care accounts for a higher percentage of health expenditure than most if not all other diseases; for most ages, dental diseases ranked second to cancer in terms of cost [30]. Treatment of caries in low-income countries by traditional restorative dentistry for the permanent dentition costs $\$ \mathrm{US} 1,618-\$ 3,513$ per 1000 children aged $6-18$ years, so the cumulative cost of treating caries in children alone exceeds the financial resources available for all health care for children in the majority of poor nations [31].

\section{An optimum intake of sugars?}

Since the first Millennium analysis of the global burden of disease, WHO's approach is to set the minimum average population level of a risk factor that minimizes a disease [32]. Thus, $115 \mathrm{mmHg}$ has been the optimum average population systolic blood level and $3.8 \mathrm{mmol} / \mathrm{l}$ the optimum total cholesterol level. This approach also led WHO to propose an intake of $<5 \mathrm{~g}$ salt/d. Taking this approach would probably mean setting the ideal goal of free (non-milk extrinsic) sugars intake, at $0 \%$. Certainly, as set out in the original analyses by WHO [33], there is no intrinsic biological need for sugar intakes as noted when those with fructose intolerance eliminate sugar intakes.

Even in children, a doubling in the burden of caries is evident on sugar intakes of $5 \% \mathrm{E}$ and the relatively new data from adult caries burdens in relation to sugar intakes in Japan and China suggest that $<3 \%$ E sugar intake is appropriate so, even given the use of fluoridated water and fluoride enriched toothpastes, sugar intakes of no more than $5 \% \mathrm{E}$ should be considered as a maximum. Levels above that will cause substantial caries burdens even with the extensive use of fluoride. Our findings support those of Moynihan and Kelly [4] whose findings from their systematic review "suggest that there may be benefit in limiting sugars to $<5 \% \mathrm{E}$ to minimize risk of dental caries throughout the life-course".
The WHO originally accepted Sreebny's proposition that $50 \mathrm{~g} / \mathrm{d}$ intake was acceptable when the DMFT in 12-year-old children was below 3 [34]. With the then high level of dental caries in children in affluent societies in the 1970s, WHO considered a goal of a mean DMFT of 3 by the Year 2000 for dental health in 12-year-olds as a reasonable target. Thus the concept of $50 \mathrm{~g}$ a day as a safe level seems to have been the basis for several countries and WHO to consider a $10 \% \mathrm{E}$ sugar intake acceptable [33]. The acceptability of a daily $50 \mathrm{~g}$ sucrose intake seemed to be reinforced by finding an increase in cavitation rates in less susceptible teeth erupted for only a year in 6-12-year-olds when sugar intakes reached nearly $10 \% \mathrm{E}$, that is, $50 \mathrm{~g} / \mathrm{d}$ [35].

The findings from this study indicate that current approaches to controlling dental caries are failing to prevent high levels of caries in adults in all countries and this relates to the current high level of sugar intake across the globe. Thus, for multiple reasons, including obesity and diabetes prevention, we need to adopt a new and radical policy of progressive sugar reduction. This is particularly appropriate given the concerns of the United Nations Environment Programme about mercury in dental amalgams and the need to "phase down" using amalgam. This means that it is now even more important to develop a radical prevention policy with a marked reduction in sugar intake since the use of fluoride on its own is insufficient to reduce substantially the burden of caries over the lifetime of individuals.

\section{Strengths and limitations of this study}

The strengths of the study include our finding that on re-evaluating the dose-response relationship between sugar and caries, we found that the sugar-caries relationship is in arithmetic terms, curvilinear and not sigmoidal and is evident at average sugar intakes well below $10 \% \mathrm{E}$. Extensive searches of the historical, as well as current literature on sugars and caries were carried out. The limitations of the study related to the absence of defined valid data on the dose-response relationship of adult caries when sugar intakes change. Therefore our findings depend on data from children together with longitudinal data on an annual basis into the fourth decade of life. However, they are reinforced by the findings of the heavy burden of dental caries in adults evident despite the widescale uses of fluorides, so caries progressively increases with age when sugar intake levels are those commonly consumed throughout the world.

\section{Article summary}

Double blind intervention trials as well as detailed biochemical and clinical studies prove that sugar intake is the major cause of dental caries whether in food or drink. The applicability of intervention trials to national 
policies is best judged by assessing the response in the population burden of caries to changes in national diets with confirmation from observations of caries prevalence in different national settings where sugar \pm fluoride intakes are known. New analyses show that the life-long burden of caries increases as sugar intakes increase from $0 \% \mathrm{E}$. The progressive accumulation of dental caries, despite widespread use of fluoride, shows that sugar intakes should be $<3 \%$ energy to minimize the disability and cost of dental caries in a population. A 5\%E sugar intake should be considered as a maximum even with the use of fluoridated water and fluoride toothpaste.

\section{Conclusions}

There is a robust log-linear relationship of caries to sugar intakes from $0 \% \mathrm{E}$ to $10 \% \mathrm{E}$ sugar. A $10 \% \mathrm{E}$ sugar intake induces a costly burden of caries. These findings imply that public health goals need to set sugar intakes ideally $<3 \% \mathrm{E}$ with $<5 \% \mathrm{E}$ as a pragmatic goal, even when fluoride is widely used. Adult as well as children's caries burdens should define the new criteria for developing goals for sugar intake.

\section{Competing interests}

There are no competing interests.

\section{Authors' contributions}

AS undertook a series of searches for national data, contacted Japanese colleagues for original Japanese papers and arranged their translation when necessary. WPTJ proposed the analytical approach, the need for additional data on adults and wrote the first draft which was then conjointly developed. Role of the funding source. No external funds were required for these analyses, interpretation or the writing of the paper. The corresponding author had full access to all the data relating to his previous cited work, sought additional data from colleagues on their data internationally and takes responsibility for the decision to submit the paper for publication. Both authors read and approved the final manuscript.

\section{Acknowledgments}

We thank Dr Shinsho Fumiaki for help in translation of some papers from Japanese, and for obtaining permission to use one of the figures.

\section{Author details}

${ }^{1}$ Emeritus Professor of Dental Public Health, Department of Epidemiology \& Public Health, University College London, London, UK. ${ }^{2}$ Honorary Professor of Nutrition, London School of Hygiene and Tropical Medicine, London, UK.

Received: 12 May 2014 Accepted: 18 August 2014

Published: 16 September 2014

\section{References}

1. Marcenes W, Kassebaum NJ, Bernabé E, Flaxman A, Naghavi M, Lopez A, Murray CJL: Global burden of oral conditions in 1990-2010: a systematic analysis. J Dent Res 2013, 92:592-597.

2. Moynihan P: The interrelationship between diet and oral health. Proc Nutr Soc 2005, 64:571-580.

3. Fejerskov O: Changing paradigms in concepts on dental caries: consequences for oral health care. Caries Res 2004, 38:182-191.

4. Moynihan P, Kelly S: Effect on caries of restricting sugars intake: systematic review to update WHO guidelines. J Dent Res 2014, 93(1):8-18.

5. Te Morenga L, Mallard S, Mann J: Dietary sugars and body weight: systematic review and meta-analyses of randomised controlled trials and cohort studies. BMJ 2013, 346:e7492.
6. Food and Agriculture Organization: Food Balance Sheets-----E_All_Data. CSV.; 2014. http://faostat3.fao.org/faostat-gateway/go/to/home/E.

7. Fisher FJ: A field survey of dental caries, periodontal disease and enamel defects in Tristan da Cunha. Br Dent J 1968, 125:447-453.

8. Sheiham A: The prevalence of dental caries in Nigerian populations. Br Dent J 1967, 123:144-148.

9. Buttner W: Zuckeraufnahme und karies. In Grundfragen der Ernahrungswissenschaft. Edited by Cremer HD. Rombach: Freiburg im Breisgau; 1971:175-191.

10. Knowles EM: The effects of enemy occupation on the dental condition of children in the Channel Islands. Mon Bull Minist Health Emerg Public Health Lab Serv 1946, (August):161-172.

11. Schulerud A: Dental Caries and Nutrition during Wartime in Norway. Oslo: Fabritius and Sonners trykkeri; 1950.

12. Jamel H, Plasschaert A, Sheiham A: Dental caries experience and availability of sugars in Iraqi children before and after the United Nations sanctions. Int Dental J 2004, 54:21-25.

13. Takeuchi M: Epidemiological study on dental caries in Japanese children, before, during and after World War II. Int Dental J 1961, 11:443-457.

14. Takeuchi M, Shimizu T, Kizu T, Eto M, Nakagawa M: Sealing of pits and fissures with resin adhesive. 4. Results of five-year field work and a method of evaluation of field work for caries prevention. Bull Tokyo Dent Coll 1971, 12:295-316.

15. Whelton H, Crowley E, O'Mullane D, Woods N, McGrath C, Kelleher V, Guiney H, Byrtek M: Oral Health of Irish Adults 2000 - 2002. Final Report April 2007. Dublin: Department of Health and Children. Brunswick Press Ltd; 2007.

16. Slade GD, Sanders AE, Do L, Roberts-Thomson K, Spencer AJ: Effects of fluoridated drinking water on dental caries in Australian adults. $J$ Dent Res 2013, 92:376-382.

17. Dye BA, Tan S, Smith V, Lewis BG, Barker LK, Thornton-Evans G, Eke PI, Beltrán-Aguilar ED, Horowitz AM, Li CH: Trends in oral health status: United States 1988-1994 and 1999-2004. Vital Health Stat 11 2007, 248:1-92.

18. Koike $\mathrm{H}$ : Studies on caries incidence in the first molar in relation to amount of sugar consumption on primary school children in Kyoto city. Bull Tokyo Dent Coll 1962, 3:44-56.

19. Okuya Y: The epidemiological study of the relation between caries incidence and sugar consumption on the second molar. Shikwa Guhuho 1960, 60:1120-1134.

20. Marthaler TM: Changes in dental caries 1953-2003. Caries Res 2004 38:173-181.

21. Bernabé $E$, Sheiham $A$ : Age, period and cohort trends in caries of permanent teeth in four developed countries. Am J Public Health 2014 104(7):115-121.

22. Hugoson A, Koch G: Thirty year trends in the prevalence and distribution of dental caries in Swedish adults (1973-2003). Swed Dent J 2008, 32:57-68.

23. Kawashita $Y$, Kitamura M, Saito T: Monitoring time-related trends in dental caries in permanent teeth in Japanese national surveys. Int Dental I 2012, 62:100-105

24. Qi X: Report of Third national survey of oral health status in China. (In Chinese). Beijing: People's Medical Publishing House; 2008.

25. Korean National Oral Health Survey. Seoul: Ministry of Health and Welfare; 2011.

26. Broadbent JM, Thomson WM, Poulton R: Trajectory patterns of dental caries experience in the permanent dentition to the fourth decade of life. J Dent Res 2008, 87:69-72.

27. Sheiham A: Sugars and dental decay. Lancet 1983, I:282-284

28. Slade GD: Epidemiology of dental pain and dental caries among children and adolescents. Community Dent Health 2001, 18:219-227.

29. Petersen PE, Bourgeois D, Ogawa H, Estupinan-Day S, Ndiaye C: The global burden of oral diseases and risks to oral health. Bull World Health Organ 2005, 83(9):661-669.

30. FDI: The Oral Health Atlas. In Mapping a Neglected Global Health Issue. Edited by Beaglehole R, Benzian H, Crail J, Mackay J. FDI World Dental Federation; 2009. http://www.fdiworldental.org/oral-health/atlas/mapping-aneglected-global-health-issue.aspx.

31. Yee R, Sheiham A: The burden of restorative dental treatment for children in third world countries. Int Dent J 2002, 52:1-9.

32. Murray CJL, Ezzati M, Lopez AD, Rodgers A, Hoorn SV: Comparative quantification of health risks: conceptual framework and methodological issues. In Comparative quantification of health Risks. Edited by Ezzati M, 
Lopez AD, Rodgers A, Murray CJL. Geneva: Oxford University Press. World Health Organization; 2004:1-38.

33. World Health Organization: Diet, nutrition and the prevention of chronic diseases, Technical Report Series 797. Geneva: WHO; 1990.

34. Sreebny LM: Sugar availability, sugar consumption and dental caries. Community Dent Oral Epidemiol 1982, 10:1-7.

35. Sheiham A: Why free sugars consumption should be below $15 \mathrm{~kg}$ per person per year in industrialised countries: the dental evidence. Br Dent $\mathrm{J}$ 1991, 171:63-65.

doi:10.1186/1471-2458-14-863

Cite this article as: Sheiham and James: A reappraisal of the quantitative relationship between sugar intake and dental caries: the need for new criteria for developing goals for sugar intake. BMC Public Health 2014 14:863.

\section{Submit your next manuscript to BioMed Central and take full advantage of:}

- Convenient online submission

- Thorough peer review

- No space constraints or color figure charges

- Immediate publication on acceptance

- Inclusion in PubMed, CAS, Scopus and Google Scholar

- Research which is freely available for redistribution 